\title{
UWB radar for detection and localization of trapped people
}

\author{
Egor Zaikov and Juergen Sachs \\ Electronic Measurement Research Lab, TU Ilmenau \\ Germany
}

\section{Motivation and literature survey}

The problem of rescuing people from beneath the collapsed buildings does not have an ultimate technical solution that would guarantee efficient detection and localization of victims. The main techniques in use are:

- Cameras with long optical fibers that are injected into the holes or fissures in the collapsed buildings (the usability of such devices and their efficiency depend on the structure of collapsed building and besides, when the victim is detected it is difficult in the most cases to determine its actual position).

- Sledge hammers are used to give a signal to potential victims, and rescuers with microphones are waiting for hearing the response (obvious limitation of this method is that unconscious people cannot be detected. Localization of victims is a problem as well)

- Search dogs are deployed in the disaster area. They detect presence of victims efficiently by smell, but information about their actual positions or quantity cannot be indicated. Moreover, dog is likely to indicate the presence of dead person which distracts rescuers from locations where living people can still be found.

Due to the ability of electromagnetic waves to penetrate through typical building materials and its significant (in order of centimetres) spatial resolution, UWB radar was considered as a prospective tool for detection and localization of people trapped beneath the collapsed building within the European project RADIOTECT (Ultra Wideband Radio application for localization of hidden people and detection of unauthorized objects). In particular, the aim was to develop a radar system that can detect person by his/her breathing through thick layer of rubble (1-2 meters of reinforced concrete). In short main RADIOTECT results related to breathing detection were presented in (Zaikov, 2010). Evidently, detection of person by respiratory activity has an added value in comparison with methods in use (listed above), since new possibilities for detection and localization of multiple unconscious victims arise.

Ability to detect respiratory and cardiac motion by means of UWB radar is reported in numerous articles and field studies. To name a few:

- In (Chernyak, 2006) basic algorithm for breathing detection in the absence of non-stationary clutter is developed and analyzed with respect to radar theory. Pulse radar is considered.

- In (Ossberger et al., 2004) algorithm for through-wall breathing detection that compensates for dilation of emitted waveform due to the wall is presented. Algorithm is 
based on Continuous Wavelet Transform. Pulse radar is considered. Besides, ability to detect breathing through $20 \mathrm{~cm}$ - thick brick wall is demonstrated.

- In (Narayanan, 2008) noise radar is used for breathing detection. Breathing signature is separated from unwanted signal components by means of Hilbert-Huang transform. Finally, breathing is clearly detected through $30 \mathrm{~cm}$ - thick concrete wall (useful signal is $3 \mathrm{~dB}$ higher then highest noise peak)

- In (Levitas, \& Matuzas, 2006) and (Levitas et al., 2008) two algorithms for breathing detection are proposed by group from Geozondas Inc. In (Levitas, \& Matuzas, 2006) breathing is detected as a strong variation in the spectrum of received radar response. However, this approach doesn't differentiate between breathing and any other motion in radar cross-section. Algorithm in (Levitas et al., 2008) detects breathing as a periodical variation of some waveform. Neither in (Levitas, \& Matuzas, 2006) nor in (Levitas et al., 2008) this waveform is considered to be known a priori in contrast to (Chernyak, 2006) and (Ossberger et al., 2004). In (Levitas et al., 2008) breathing was clearly detected through 16-cm thick brick wall by means of impulse radar. Importantly, ability to detect two breathing persons simultaneously is demonstrated. Breathing rate of two persons was deliberately different, but this difference is not the only distinction between two persons in received signal since it can be seen that two breathing signatures arrive at different distances to antennas.

Historically, UWB radars were extensively applied as Ground Penetrating Radars (GPR) in the fields of geophysics, archaeology and for the detection of buried mines prior to using them for the problem described in this chapter. No surprise though that some investigations were carried out in order to determine the efficiency of GPRs for the detection of people buried beneath the rubble:

- Thorough test of GPR as a detector of living person is described in (Bechtel Special Technologies Laboratory Ground Penetrating Radar, 2003). The GPR was originally developed under the sponsorship of the U.S. Department of Energy's Special Technologies Program for other applications (unfortunately, no details about antenna type/bandwidth is given in the report). This Operational Test and Evaluation was conducted by personnel of Virginia Task Force 1 (VATF1), Urban Search and Rescue (US\&R) Team and the California Task Force 2 (CATF2) US\&R Team. Test was conducted on November 5, 2003, at the rubble pile of Fairfax County. To cite from the report, 'In general, the system has the capability to penetrate 1-2 feet of rubble and an associated airspace of up to 6 feet. In some scenarios, the system detected breathing of victims up to 9 feet through one or two thicknesses of concrete and airspace'

- More results about using commercial GPR (SIR-3000 by Geophysical Survey Systems, Inc.) are presented in (Geophysical Survey Systems, Inc., 2005). Importantly, antennas recommended for breathing detection are $270 \mathrm{MHz}$ or $400 \mathrm{MHz}$ antennas (lower frequencies then in the most of other studies). To cite from the report, 'GPR can easily penetrate the concrete debris and "bend around" the metal reinforcing bars. Tests have also shown that GPR can note the presence/absence of a live person behind an intact $50 \mathrm{~cm}$ heavily reinforced (2 mats of rebar) wall.'

- Last but not least, there is a commercially available device for detecting people beneath the rubble: LifeLocator ${ }^{\mathrm{TM}}$ by UltraVision Security Systems, Inc. Citation from (UltraVision Security Systems, Inc.): 'Detection Distance through Debris Pile: Up to 15' (4.6 meters) for breathing, up to $20^{\prime}$ (6.4 meters) for motion'. Rubble type is not specified. 
Separately I should briefly mention the use of narrowband radars for the problem under investigation.

- In (Arai, 2001) breathing person was detected through more then 2 meters of diverse debris.

- Commercial Bioradar BR402 by BOS - Sondermaschinenbau $\mathrm{GmbH}$ is a narrowband device with operating frequency $1299 \mathrm{MHz}$. Remarkable penetration depth is mentioned (however, without specifying material type and airspace size): 'The coverage depends on the antenna shape and the material to be penetrated, and can be up to 8 meters', (BOS Sondermaschinenbau $\mathrm{GmbH}$ ).

Finally, the contents of previous research in the area determined the direction of our approach to breathing detection considerably. Below relevant points related to our effort in connection to earlier studies are summarized:

- It can be seen that reported depth were breathing is detected with narrowband radar is higher then that for UWB radars. Obviously, this can be explained by notion that radar electronics in the case of narrowband device is able to treat signals of higher power. However, narrowband radars have poor resolution capacity and that is, they do not provide sufficient abilities to detect multiple persons or, most importantly, to determine the position of trapped victim. Besides, there are much more possibilities for cancellation of clutter arising from moving surrounding in UWB radar. Our aim was to utilize unique abovementioned properties of UWB radar.

- Previously reported research in breathing detection with UWB radar was mainly concentrated on detection and enhancement of useful signal. While viable data processing methods proposed are diverse, according to (Yarovoy \& Ligthart, 2007), the fact that UWB radar allows for positioning of trapped victim have not yet been proven experimentally. The problems of multiple victims and clutter did not receive much attention as well.

\section{Hardware for breathing-detecting radar system}

Prototype radar developed for through-rubble detection of human being is a M-sequence radar with one transmitting and two receiving channels (Zaikov et al., 2008). Its structure is shown if figure 1.

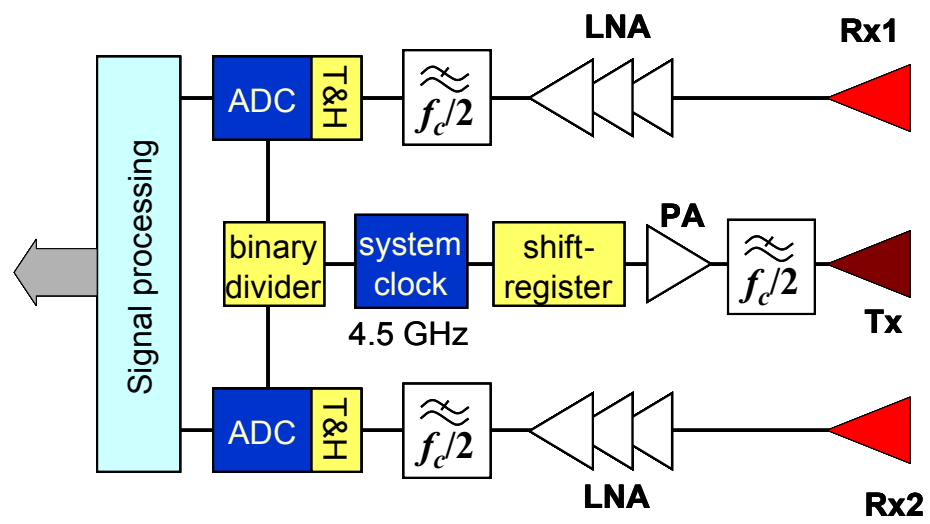

Fig. 1. System structure an M-Sequence radar with one transmitter and two receivers. 

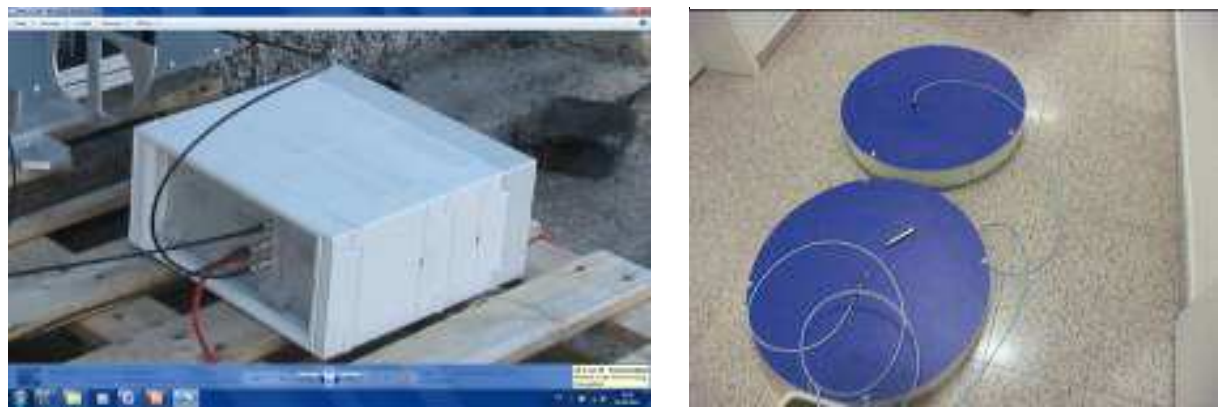

Fig. 2. Radar device and antennas used in prototype system. Courtesy to MEODAT, Ilmenau and IRK, Dresden.

The shift-register, pulsed by the RF-clock, provides the M-sequence, which is a stimulus signal transmitted by the Tx-antenna. Further, on the receiver side signal is converted into the digital domain via sub-sampling controlled by binary divider. Obviously, the received signal is a pseudo-random one, and it cannot be further used directly. Thus, the time-domain radar signal is reconstructed by means of a correlation procedure. After that, the signal is available for further processing in the form similar to that of the impulse radar signals.

The system clock frequency for the device is about $4.5 \mathrm{GHz}$, which results in the operational bandwidth of about DC-2.25 GHz. The M-sequence order is 9, i.e. the impulse response covers 511 samples regularly spread over $114 \mathrm{~ns}$. This corresponds to an observation window of $114 \mathrm{~ns}$ leading to an unambiguous range of about $16 \mathrm{~m}$ in free space. 256 hardware averages are always computed within FPGA of the radar head to provide a reasonable data throughput and to improve the SNR by $24 \mathrm{~dB}$. Additional software averaging can be completed by main computer, if required.

Advantages of using M-sequence radar module as related to the topic of this chapter are briefly summarized below:

- Low jitter due to the stability of shift register and binary divider. Importance of this point for breathing detection is explained in part 3 of this chapter.

- Fast data acquisition. The system works reliably while collecting 32 A-scans per second which is certainly fast enough to catch the fastest human breathing. Moreover, this acquisition rate gives us good possibilities to discriminate breathing response from unwanted clutter like drift of electronics and reflection from moving surrounding.

- Lowest frequency in radar response is a few MHz. Although motion associated with respiratory activity is minor, frequencies below $300 \mathrm{MHz}$ are still useful for breathing detection in many cases(less efficient then higher frequencies if we do not take rubble attenuation into account though due to the minor magnitude of breathing in comparison with wavelength). Ability of electromagnetic waves to penetrate typical building materials is higher for low frequencies. Measurement example in support of this point in the case of breathing is given in figure 3 .

The last point about frequency range was crucial for constructing the antennas to be used in radar prototype. One important requirement for the total system is that it has to be efficient under diverse conditions on different types of rubble, including moist rubble. In practice 
that means retaining low frequencies well below $300 \mathrm{MHz}$ in the measured data. On another hand the lower the frequencies the larger the antennas to be used. As a compromise between the size of antennas (smaller antennas mean less deployment time, they are more convenient) and the need to catch low frequencies, planar spiral antennas $70 \mathrm{~cm}$ in diameter were chosen (figure 2). Transmitting and receiving antennas have different polarization in order to minimize the crosstalk between antennas and increase the transmitted power which gives us possibility to penetrate more rubble. Antennas are functional in the frequency range from $0.15 \mathrm{GHz}$ to $1.1 \mathrm{GHz}$.
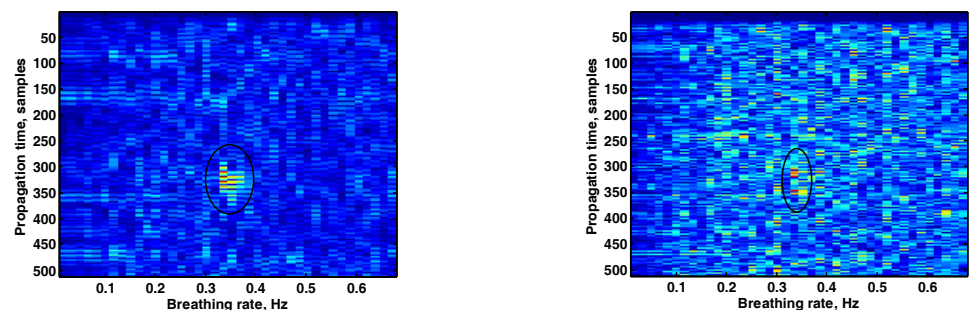

Fig. 3. Person, breathing beneath the heap of bricks inside the pipe, made of reinforced concrete. Data is filtered with upper cutoff frequency of $300 \mathrm{MHz}$ (on the left, person is detected as bright red point and marked) and $700 \mathrm{MHz}$ (on the right, location of person is marked, but it is not distinguishable from noise peaks).

\section{Statement of the problem and breathing detection}

Breathing signature in radar response is caused by the minor (in comparison to walking) shift of body parts. The physical problem of detecting such minor motion is illustrated in figure 4 .

It can be observed that minor motion, like breathing, is felt mainly on the flank of the measured signal, since the slight shift of the waveform from its incipient state produces the largest variation of waveform at the steepest slope. This is described by the following relation:

$$
\Delta V=a \frac{2 \Delta d}{c}
$$

where $a$ is the slope and $c$ is speed of light.

Therefore, the smallest displacement $\Delta d$ which can be observed depends on the rise time of the backscattered signal and the smallest detectable voltage variation $\Delta V$. The rise time of the waveform is typically limited by the test object (i.e. the rubble or the wall) if the radar bandwidth is sufficiently high as it is in our case. The voltage resolution is limited by random noise caused by the radar device or external interferer. In what follow, we suppose that no external interferer is present.

In that case, the total noise on the flank results from additive noise and jitter which can be expressed by following equation:

$$
\sigma=\sqrt{\sigma_{a}^{2}+a^{2} t^{2}}
$$


where $\sigma_{a}$ is additive noise, $t_{j}$ is rms jitter and $a$ is the slope the signal interval of interest.

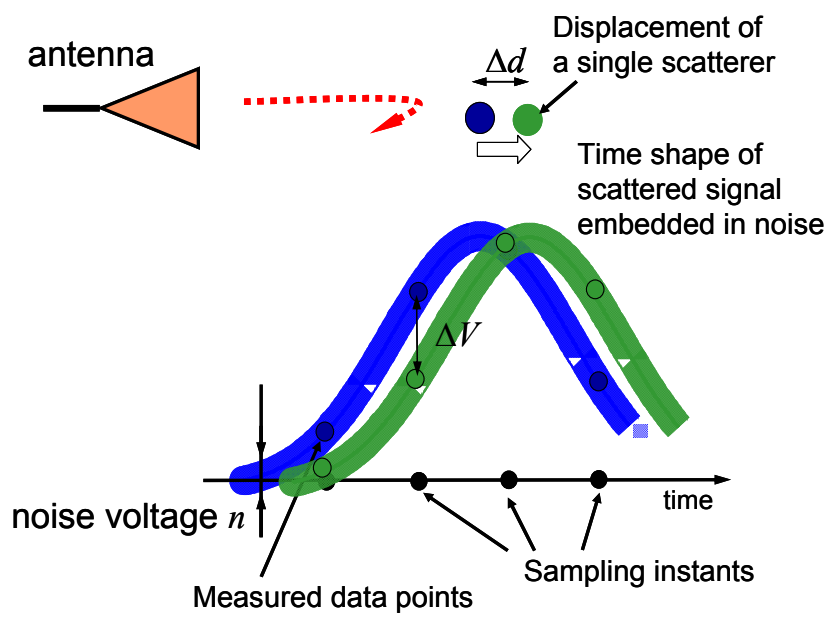

Fig. 4. Principle of detecting minor motions by means of UWB radar

That is, the jitter performance of the radar device is crucial for this particular task, since both jitter and minor motion caused by breathing are originated at the flank of the radar signal. However, measurements were carried out to estimate the jitter in M-sequence radar and only additive noise could be observed. As it was mentioned above, for our problem this is one of the most important advantages of an M-Sequence conception compared to other ultra-wideband principles.

Breathing manifests itself in the radar response as a very specific signal. Below, its features are summarized that were taken into account during development of detecting processing methods (see figure 5 for illustration of these points):

1. The geometrical variations of the chest caused by breathing will be quite less than the range resolution of the radar. This can be observed in figure 5, where response from breathing person is shown at different phases of respiratory activity.

2. Distance from antennas to breathing person does not change during the measurement (otherwise, the change is indicated by strong motion). That is, breathing typically appears at the certain moments of propagation time (see figure 5).

3. Breathing can be considered as periodical motion over a certain interval of time. The frequency of breathing can change slowly with the time, but it is always within the frequency window, which is known a priori $(0.2-0.5 \mathrm{~Hz})$. In figure 5 the periodical patterns, produced by breathing are shown.

4. Breathing appears as correlated motion in several neighbouring cells in radar response. The size of the breathing-related segment of radar cross-section is determined by antenna, physical size of the body which is moved during the respiration activity, position of the body, by rubble type, thickness and structure.

5. The response from breathing person can be extremely weak, since the victims of interest are buried beneath the rubble which strongly attenuates the sounding waves. 


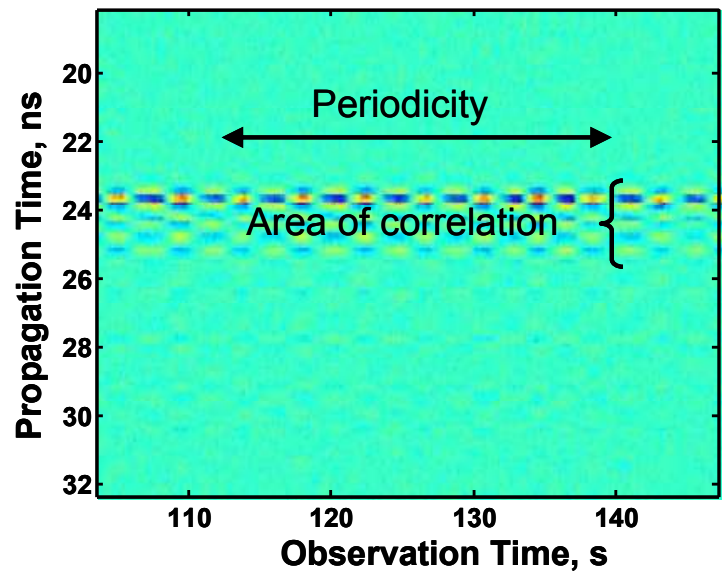

Fig. 5. Person, breathing 2 meters away from the antennas.

Second and third point lead to the idea of using time-frequency representations (in slowtime) for breathing detection similar to how it is done in (Narayanan, 2008) and (BOS Sondermaschinenbau $\mathrm{GmbH}$ ) in order to discriminate breathing from any other signal component. However, in this work we mainly concentrate on the situation when point four from the list is valid. In this case instantaneous amplitude of breathing is small in comparison with noise and it is not well detected in time-frequency representation while it is good visible in frequency after appropriate signal processing.

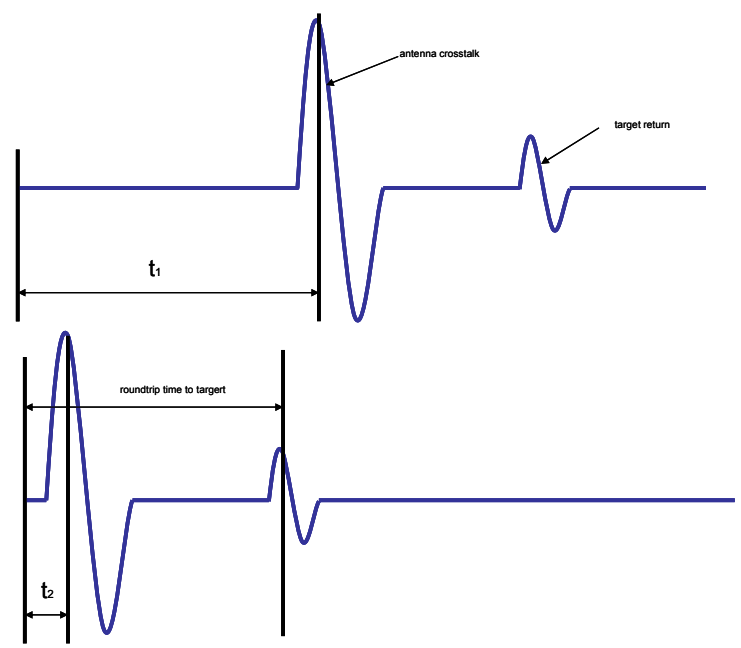

Fig. 6. Shift of IRF to time-zero estimate.

In M-sequence radar used IRFs are shifted by random value in propagation time every time the device is switched on. That is, information about distance from antennas to object is not related to propagation time where the motion is seen in the raw data. To avoid this effect, 
IRF can be circularly shifted left by $t_{1}-t_{2}$ (see figure 6), where $t_{1}$ is a time instant where maximal value of IRF arises (it is supposed to be related to antenna crosstalk) and $t_{2}=\sqrt{\varepsilon} d / c$, where $\varepsilon$ is a dielectric constant of rubble and $d$ is a distance between the centres of transmitting and receiving antennas.

Antenna cross-talk and multiple responses from stationary background typically dominate the radargram, hampering straightforward motion detection. That is, the stage of background removal algorithm should be implemented within the software for motion detection. Due to our a priori knowledge about breathing frequencies, the task of background removal is reduced to high-pass filtering in the direction of observation time without taking into account relocation of the target. However, in one of the algorithms proposed in the chapter for non-stationary clutter reduction, signal variation that is slower then lowest breathing rate possible is used for estimating clutter. That is, the cut-off frequency used for high-pass filtering at this stage should be quite lower then $0.2 \mathrm{~Hz}$. In addition, vertical (fast-time) filtering should be carried out to limit the bandwidth to the actual bandwidth of received signal. In our case that means bandpass FIR filtering in the range from $150 \mathrm{MHz}$ to $1.1 \mathrm{GHz}$ (this corresponds to antenna bandwidth)

\subsection{Localization of useful signal in the observation time direction}

If the signal after pre-processing (shifting to time zero and band pass filtering) is denoted as $h(t, \tau)$, it is convenient to transform the signal into frequency domain $H(t, f)$. This allows us to operate over the signal in the domain, where breathing is localized most compactly due to its periodicity, although breathing is still spread over a certain range in propagation time (see figure 5). From the point of view of detection theory, the absolute value of $H(t, f)$ computed via FFT is the optimal statistics for detecting the sine component with frequency $f$ and uniformly distributed phase appearing at the distance, corresponding to propagation time $t$. Besides, phase of $H(t, f)$ is also important for the target signature enhancement (see next chapter). In practice, $(t, f)$ is the most convenient domain both for signal enhancement and for final decision about whether the person is present.

Experiments prove that for considerable time interval signal from breathing person can be approximated by sine quite precisely. However, sine is not perfect and some part of energy is spread over the bandwidth and besides, the frequency of breathing can change with the observation time going. One example when breathing is not detected via FFT due to its nonstationarity is given in figure 7 .

In order to get more consistent estimate of quasi-periodical breathing response we used method similar to well-known Welch technique (given below). The main difference of our approach from this classical method is that we do not average periodograms of data segments. Instead, we use FFTs of each segment for calculating estimates via crosscorrelation method described in the next part and then average results. Another important aspect is the size of segments for computing FFT. Our chose is to use one--two minutesegments: this is close to the time of data acquisition for breathing detection as a periodical signal via FFT given in literature. Of course, nobody can say in advance how breathing signal of particular person will change over time, but this value seems to produce good results in our measurements (figure 7). 

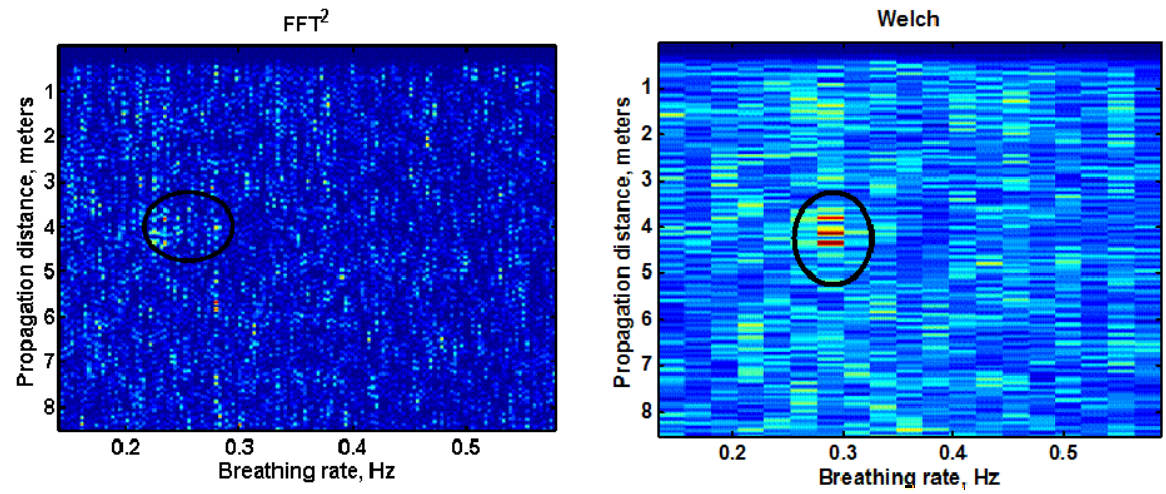

Fig. 7. Person breathing about 1 meter deep beneath moist rubble as detected by two methods.

\section{Welch algorithm:}

1. Each signal $h\left(t_{i}, \tau\right)$ is divided into the number of overlapping signals.

2. Periodogram of each segment is calculated as $S_{i, k}\left(e^{j \omega}\right)=\frac{1}{n}\left|\sum_{l=1}^{n} w_{l} h\left(t_{i}, \tau_{l}\right) e^{-j \omega l}\right| / \frac{1}{n} \sum_{l=1}^{n}\left|w_{l}\right|$, where $n$ is length of the segment and $k$ is its number, $w$ is a windowing function.

3. The set of periodograms is averaged over $k$ in order to calculate PSD estimate $S_{W E L C H}\left(e^{j \omega}\right)$

\subsection{Enhancement of useful signal in the propagation time direction}

As it was mentioned above, any motion caused by breathing arrives at several neighbouring instants in propagation time. However, backscattered waveform is not known a priori, since it depends on antennas, the size of the body during breathing, body position, rubble thickness, structure and its dielectric properties.

Similarity between two signals can be measured by means of cross-correlation. Thus, since both coherence and energy of signals increases this measure, cross-correlation can be used for breathing detection in a way, described below.

In slow-time direction cross-correlation of two datasets with different fast time $t_{k}$ indices at frequencies $f_{k}$ is easily calculated in $(t, f)$ domain:

$$
R_{i, j, k}=\operatorname{real}\left(H\left(t_{i}, f_{k}\right) H^{*}\left(t_{j,} f_{k}\right)\right)
$$


For the given absolute values of $H\left(t_{i}, f_{k}\right)$ and $H\left(t_{j}, f_{k}\right)$, absolute value of $R_{i, j, k}$ is maximal when the phase-shift between periodicals, represented by $H\left(t_{i}, f_{k}\right)$ and $H\left(t_{j}, f_{k}\right)$ is either zero or $\pi$ (that is, periodicals are either in phase or maximum of the first periodical corresponds to the minimum of the second one). These two kinds of phaseshifts exist between breathing-related signal of two distinct types (see figure 5). Breathing response can be enhanced by averaging $k$ maximal absolute values of cross-correlation terms within the $n$ consecutive cells in the direction of propagation time.

\section{Non-stationary clutter reduction}

Electromagnetic waves are radiated by UWB antennas in all directions. Of course, most of the energy is directed towards rubble heap under investigation, but given the weakness of useful signal due to the rubble attenuation reflections from non-stationary background can hamper detection of breathing victims significantly. In general, this is valid for any scenario where moving objects are present in the vicinity of antennas (distance of few meters in our case). Typical sources of non-stationary clutter are: trees and shrubs or metallic rebars when the weather is windy; people passing by the place of operation, trucks working in the area.
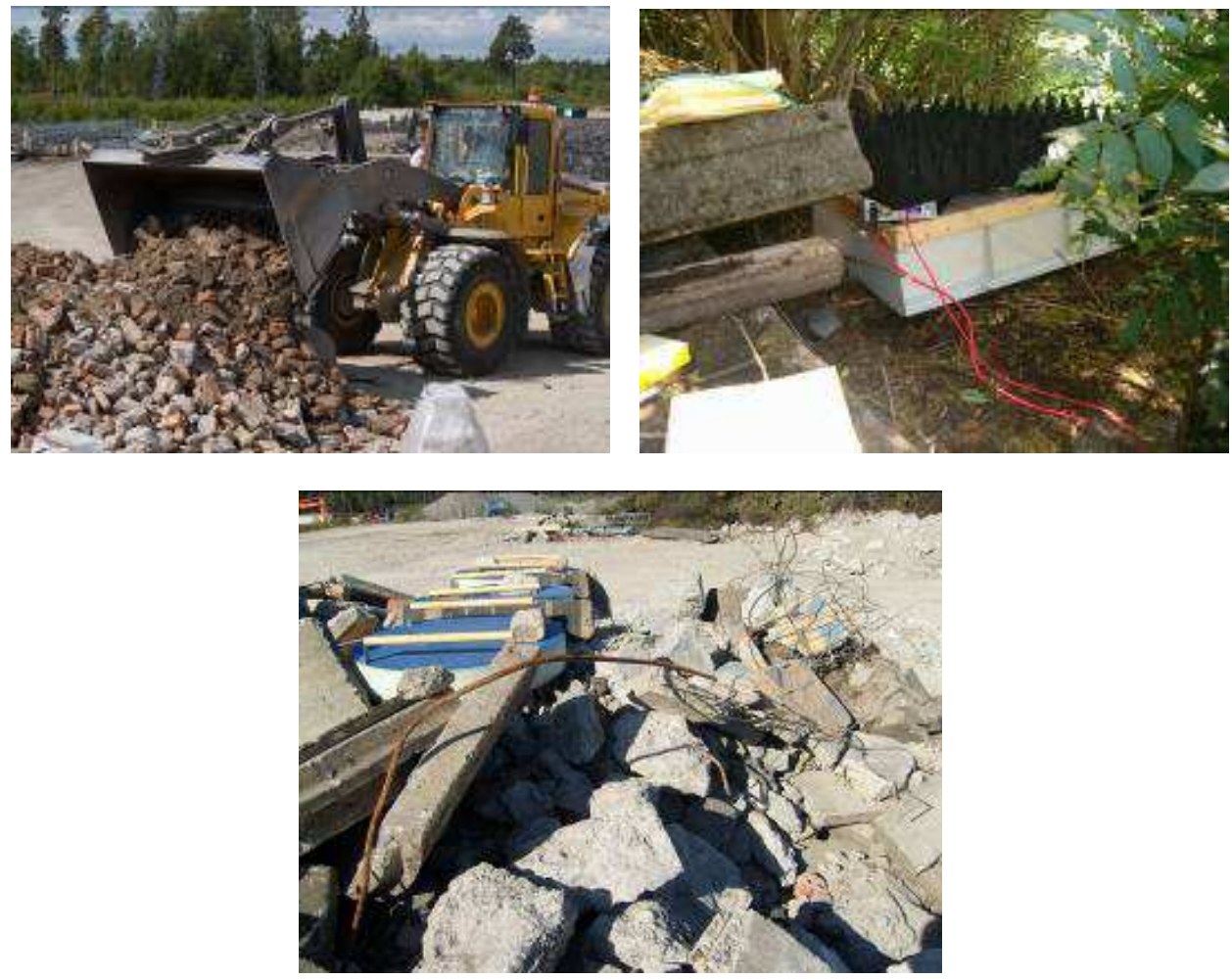

Fig. 8. Sources of non-stationary clutter at measurement place 
Evidently, some steps can be carried out to prevent clutter from handicapping the measured data by removing all its sources from the area, but this requires significant time and manpower. Besides, some measures can be taken with using metal covering and absorbing materials in order to alleviate the problem. This helps to a certain extent, but none absorber is ideal and the lower the frequency we are working with the more it is difficult to shield it, especially given that as such system should be mobile and easy to handle. That is, the problem of reducing the non-stationary clutter with appropriate software methods had to be addressed. The problem of clutter removal is complex from algorithmic point of view because there is not much a priori information about it which could serve as a basis for solution. Clutter can overlap with breathing signature in distance, appearing in the same frequency range. The problem is similar to characterizing moving environment for video cameras and in both these problems there seems to be no ideal solution and diverse algorithms are being developed in this field. Further in this chapter we consider two strategies we used to solve the problem and ideas behind them.

\subsection{Signal-Clutter separation with Principal component analysis (PCA)}

Principal component analysis (PCA) is a data processing tool, frequently used in image processing, data compressing and data visualization. PCA reveals the orthogonal basis of vectors (principal components) with a specific property, that projection of original observations on the first principal component contains the largest variance possible (first vector is chosen in such a way, that the variance in the projection is maximal). The most popular task for using PCA is dimensionality reduction in a data set by retaining those characteristics of the dataset that contribute most to its variance, by keeping lower-order principal components and ignoring higher-order ones.

Mathematically, the basic operation for computing PCA is singular value decomposition (SVD) of the data matrix:

$$
H=U D T^{T}=\sum_{i=1}^{N} u_{i} D_{i, j} v_{i}^{T}
$$

where $V=\left[v_{1}, v_{2}, \ldots, v_{N}\right]$ and $U=\left[u_{1}, u_{2}, \ldots, u_{N}\right]$. SVD calculates $N$ uncorrelated sequences, PCs $y_{i}=D_{i, i} v_{i} . D$ is a diagonal matrix of singular values, and $D_{i, i}$ decrease for larger $i$. Both PCs $y_{i}$ and eigenvectors $u_{i}$ often have simple graphical interpretation, depending on the nature of input data. For instance, in GPR data first PCs often represent clutter component, since largest variance in observations comes from the ground return which is slightly different for the data acquired at different points. In this case, there are strong peaks in first $y_{i}$ at the distance of ground return and first $y_{i}$ shows how ground return changes with the position of measurement.

With respect to our problem we can expect that after applying SVD to $h(t, \tau)$ breathing will be confined to different PCs than clutter, since breathing and clutter are uncorrelated types of motion. Notably, this is the only a priori assumption about the measured data used in this method. Further processing is reduced to projecting the measured data onto selected 
PCs $y_{i}$ that contribute significant portion of energy into measured dataset and making decision, whether some of them represent breathing (this decision can be made on periodicity of useful signal). In case only noise is present in the measured data (no nonstationary clutter) ideally breathing should be confined to the PC responsible for largest variance $y_{1}$. In the cluttered media first few PCs typically represent clutter, which is stronger then attenuated breathing signature.

In practice, PCA yields remarkable separation between signal and clutter for many scenarios. However, the more frequently clutter motion arises in the data, the less it seems to be 'uncorrelated' with breathing and the worse they are separated.

\subsection{Estimating the clutter from low-frequency variation}

As it was mentioned before, we can assume that breathing signal cannot have rate less then certain value (we use $f_{\bar{l}}=0.2 \mathrm{~Hz}$ ). On another hand, in practice we do not have clutter sources fluctuating so fast that data content with rates slower then $f_{l}$ is not disturbed, any response from walking person or wavering plants has strong low-frequency component. Based on this a priori assumption following simple algorithm for clutter cancellation can be used:

1. Two datasets are calculated: $h_{\text {fast }}(t, \tau)$ and $h_{\text {slow }}(t, \tau)$ from original $h(t, \tau)$ by means of high-pass filtering and low-pass filtering accordingly. Cut-off frequency equals $f_{l}$

2. If at certain instant $h_{\text {slow }}\left(t_{i}, \tau_{j}\right)>k \sigma_{\text {slow }}$, then $h_{\text {fast }}\left(t_{i}, \tau_{j}\right)$ is considered to be cluttered and it is cancelled. $\sigma_{\text {slow }}$ is a standard deviation of $h_{\text {slow }}(t, \tau)$ measured in clutter-free environment. Typical value for $k$ is in the range $3-3.5$. That is, the clutter is detected when in $h_{\text {slow }}(t, \tau)$ we receive value which is unlikely to be generated by noise.

Obvious advantage of this algorithm is that separation of clutter from useful data is not blind, since clutter is detected in $h_{\text {slow }}(t, \tau)$ where breathing signal cannot appear.
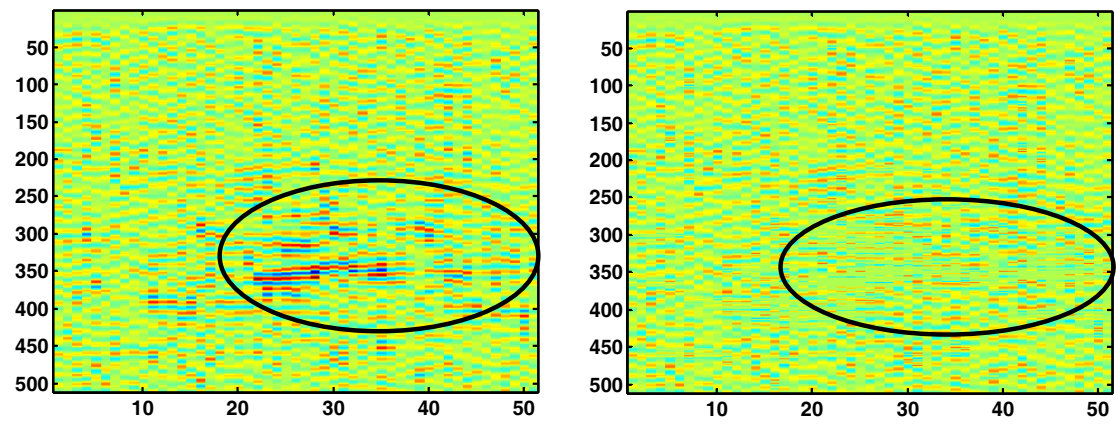

Fig. 9. $h_{\text {fast }}(t, \tau)$ before and after clutter cancellation Few results for clutter cancellation are shown below. 

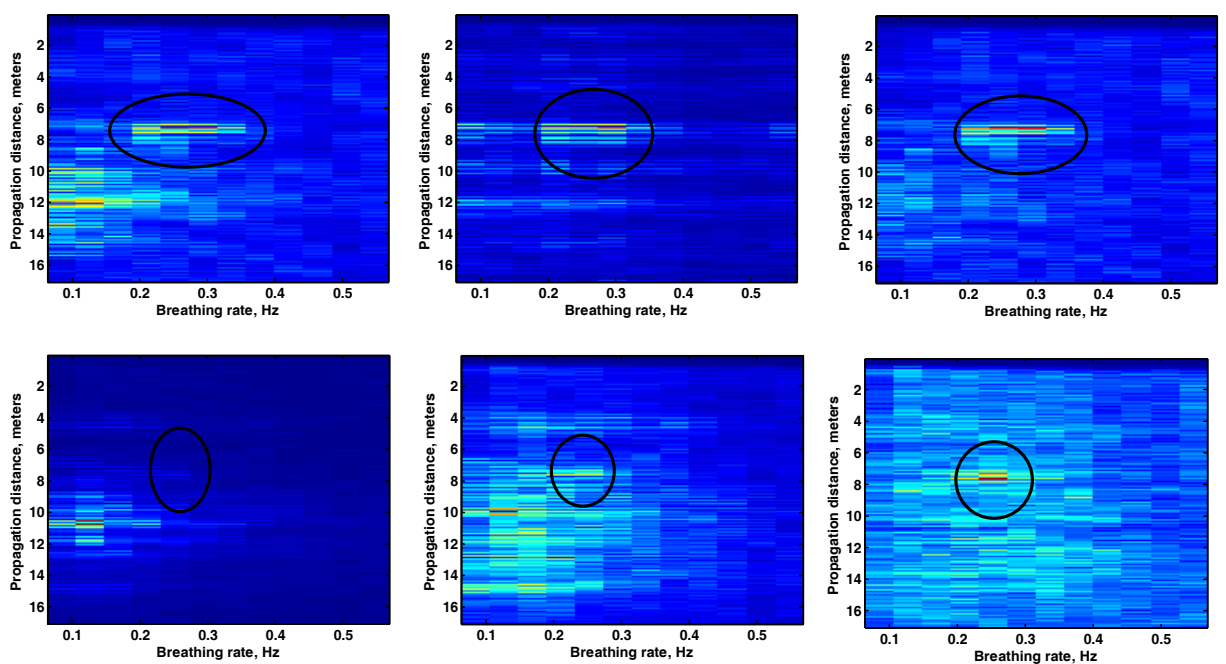

Fig. 10. Breathing person in radar data before clutter cancellation (left), after processing with PCA (the number of components to represent breathing s chosen as a best feat by operator) and after clutter cancellation from low-frequency variation (right) for two different scenarios

However, it should be noted that although both algorithms described are functional, there are much more strategies possible due to the complexity of the problem.

\section{Localization of breathing person}

Localization of breathing person is the final stage of data processing applied to the problem under discussion. This stage incorporates most of the added value of system in total, since while there are multiple methods for detecting trapped victims (search dogs is probably most successful one), finding out the position of person is much more difficult with present techniques.

With one transmitter, number of receiving antennas necessary to localize a person is three. However, it is convenient to consider main aspects of the problem for $2 \mathrm{~d}$ space, two receiving antennas as it is shown in figure 11 and then extend it to $3 \mathrm{~d}$ and three receivers. Antenna configuration shown in figure 11 reflects antenna system of radar prototype created in RADIOTECT project and most of the data was collected with person right beneath the antennas. For $3 \mathrm{~d}$ localization additional measurement with relocating receiving antenna from initial position was carried out.

In radar response electromagnetic waves reflected from different points in space arrive at different moments in fast time, which is related to distance passed by waves. We consider rubble as a homogeneous media, since no information about its internal structure is available. Although in our experiments on real rubble no significant effects of its heterogeneity spoiled localization results, this is likely to limit system performance for some scenarios. In homogeneous media distance passed by electromagnetic waves between transmitter, $i$-th receiver and reflected from some object equals 


$$
l_{i}=\frac{c t}{\sqrt{\varepsilon}}
$$

Set of possible Cartesian coordinates for an object appearing in radar at such distance for scenario in figure 11 is given by following equations:

$$
\begin{aligned}
& l_{1}=\sqrt{x^{2}+y^{2}}+\sqrt{(x-d)^{2}+y^{2}} \\
& l_{2}=\sqrt{x^{2}+y^{2}}+\sqrt{(x+d)^{2}+y^{2}}
\end{aligned}
$$

Each equation describes elliptical curve around antennas different for each point in radar response. When we find a point beneath antennas where two ellipses corresponding to one object cross, we estimate position of this object via calculating Time of Arrival (TOA) of electromagnetic waves.

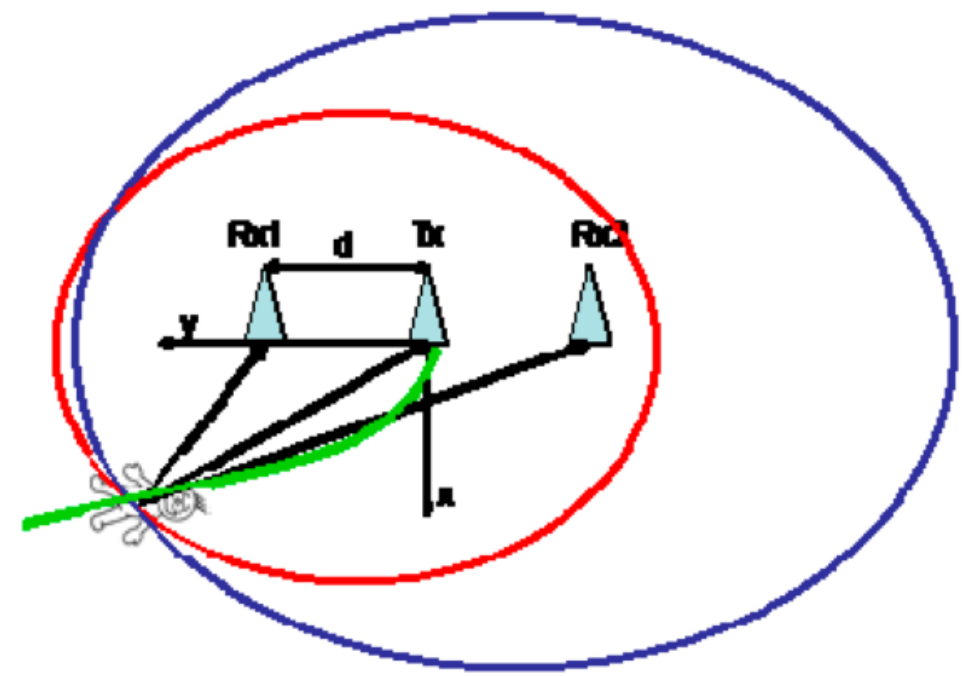

Fig. 11. Localization principle

Another possible way is to calculate Time Difference of Arrival (TDOA) of the object response in two receiving channels via

$$
l_{1,2}=\sqrt{(x+d)^{2}+y^{2}}-\sqrt{(x-d)^{2}+y^{2}}
$$

It can be seen in figure 12 that there is certain shift of breathing response in one channel relative to the second one in fast time. For each such shift there is a hyperbolic curve in the space (shown green in figure 11), representing all possible points where reflecting object can be situated. Equation (8) determines this hyperbola. Finally, for given antenna configuration 
it is possible to calculate position of the object using combined TOA/TDOA estimate (finding crossing of ellipse and hyperbola). In this case, for calculating the size of this shift maximizing the average cross correlation (as in 3.2) from dataset composed of both receiving channels with respect to size of the shift was carried out. Often using combined TOA/TDOA estimate gives less angular error in localization rather then pure TOA (it can be seen in figure 13 that crossing of ellipse and hyperbola is less blurred around the antennas). Another interesting point in figure 12 is that waveforms associated with breathing look pretty different for two identical receiving antennas even though they are not separated by rubble heap from breathing person.
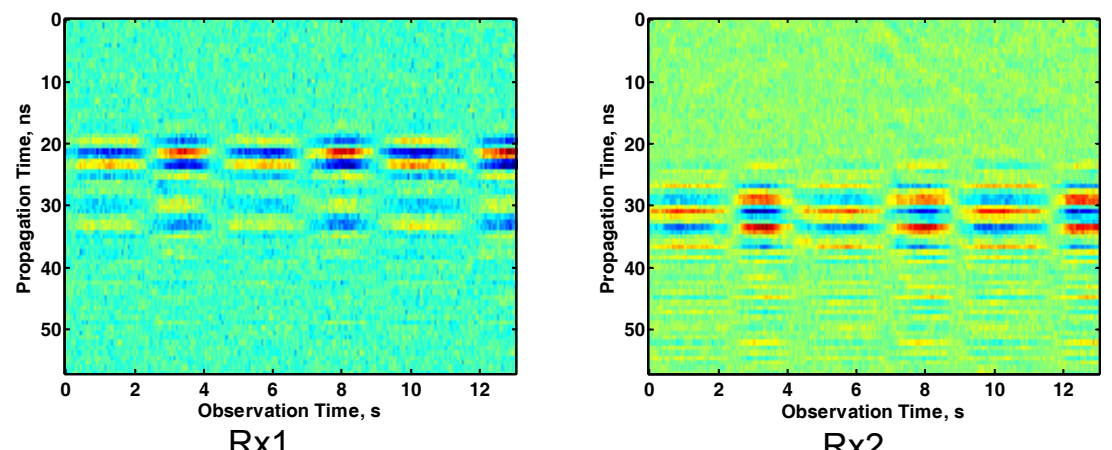

$\mathrm{Rx} 2$

Fig. 12. Breathing person as seen by 2 radar receivers.
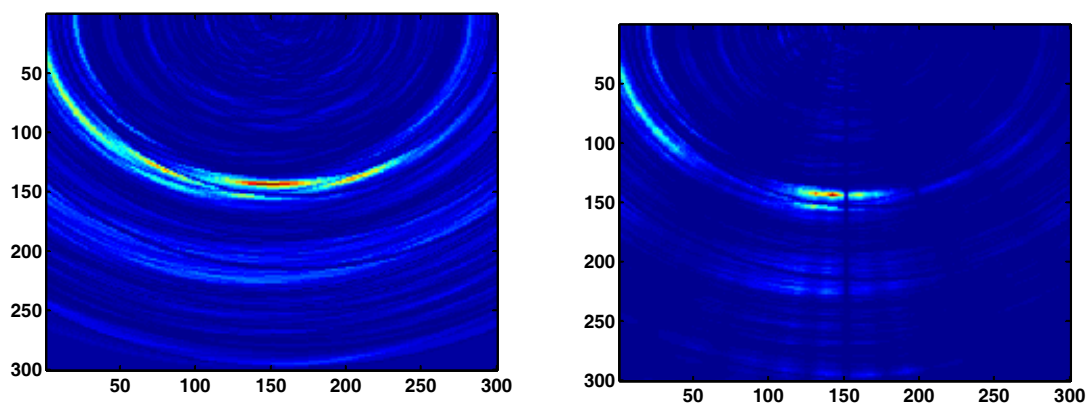

Fig. 13. Object seen by radar localized via TOA method (left) and combined TOA/TDOA approach (right)

Finally, algorithm used localization of breathing person is described below:

1. Detecting breathing signature and its rate in input data $H(t, f)$ by average crosscorrelation method. For both receiving channels vectors $H\left(l_{i}, f_{j}\right)$ are calculated.

2. Calculating "geometry" matrices, containing possible distances were breathing person can be situated. Matrices contain all possible distances correspondent to TOA and TDOA estimates. 
3. Interpolation of each $H\left(l_{i}, f_{j}\right)$ and their local average cross-correlations onto appropriate $2 \mathrm{~d}$ "geometry" matrix and addition (multiplication) of the results to receive image $I(x, y)$.

For $3 \mathrm{~d}$ localization and three receiving antennas almost identical approach was used with TOA estimates. The only modification is extension of "geometry" matrices to $3 \mathrm{~d}$.

Algorithm has following limitations:

- Heterogeneity of rubble can cause serious mistakes in some scenarios

- Imprecise value of $\varepsilon$ for rubble material under real conditions can cause some error in localization.

- In the case of two or more victims if they are situated at almost identical distances to antennas ghost target can appear (this happens because ellipses related to different victims cross). However, all victims in this case will be shown.

\section{Experimental results}

In this part measurement results are summarized excluding those presented above in order to clarify some aspects of signal processing.

\subsection{Breathing detection in intact building}

Any can assume that after building is collapsed in some places thickness of ruins to penetrate will be less then in other locations. However, in average detection capacity is expected to be better in collapsed building, since due to the smaller distance between antennas and person propagation losses decrease. In figure 14 breathing person is detected as a yellow spot through three floors of intact building.
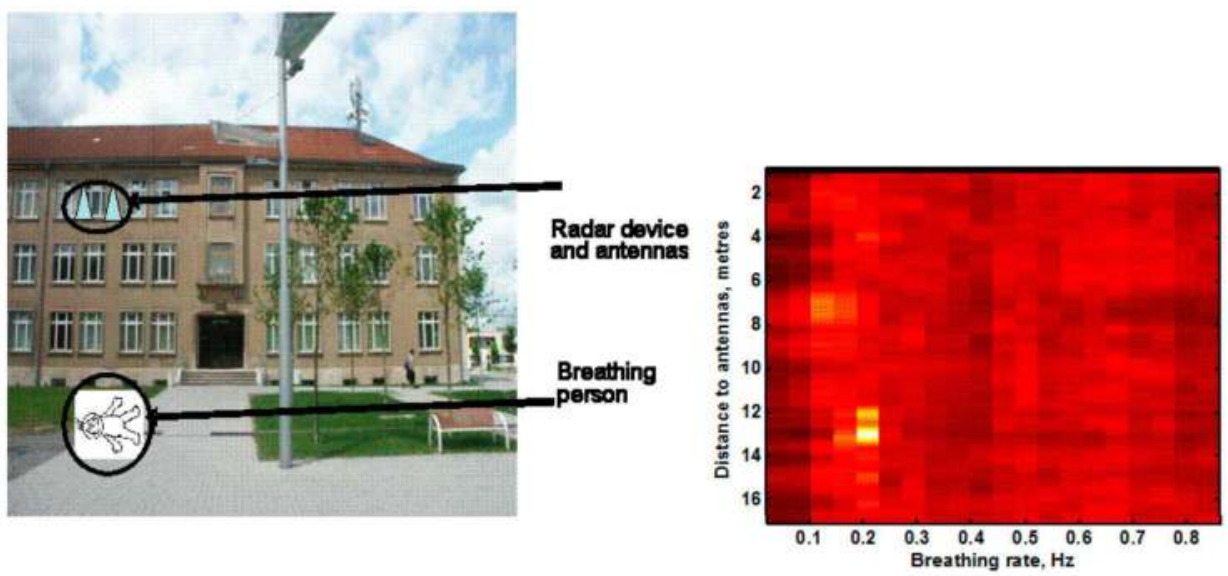

Fig. 14. Detection of breathing person in intact building. Person was lying on the floor in the basement, while radar device and antennas were on the second floor 


\subsection{Breathing detection through the heap of rubble}

Most of other measurements were carried out on the pile of diverse rubble (mainly composed of reinforced concrete). During measurements campaign thickness of rubble was increased from 1.2--1.3 up to 1.6-1.7 meters, which depth (as it can be seen in figures below) produced result on the limit of our detecting capabilities. Detection through 1.2-1.3 meters is evident (although as it can be seen some positions and people produce better result then others). Besides, tests with multiple persons (up to three) were carried out.

Detection methods described above are online and they were implemented inside GUI, operating the radar device. Thus, all experimental results in this part represent screenshots taken from the measurement software immediately at the test area. Breathing is detected as a bright point in the left part of the screen which shows data in $(t, f)$-domain. Right part of the screen represents preprocessed data in $(t, f)$ and can be used for detecting strong motion.
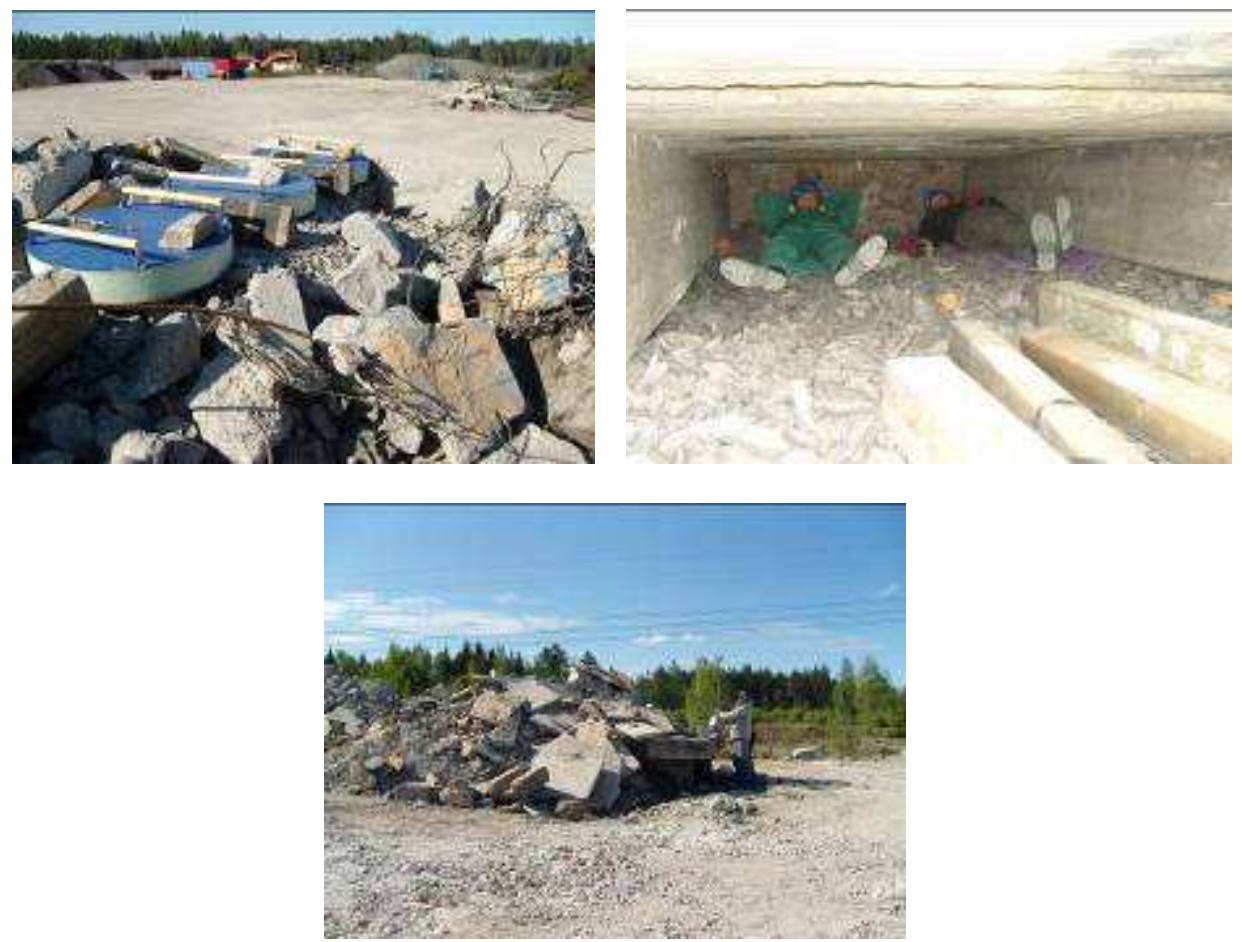

Fig. 15. Detection area 


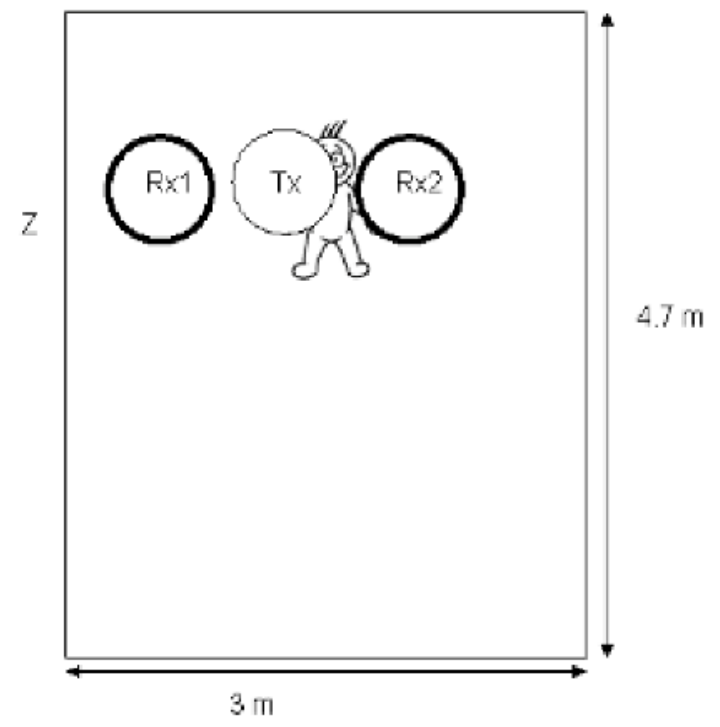

Fig. 16. Scenario for establishing penetration capabilities of the radar, view from above

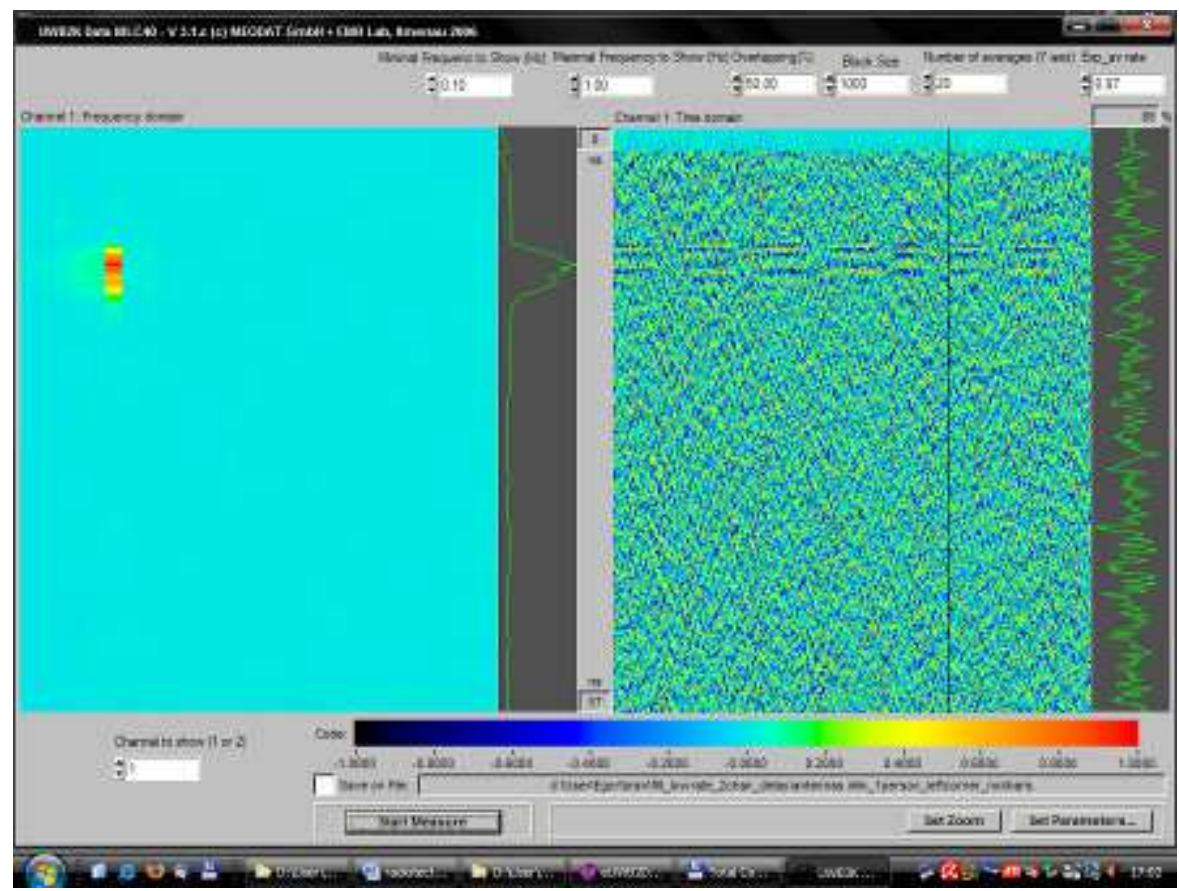




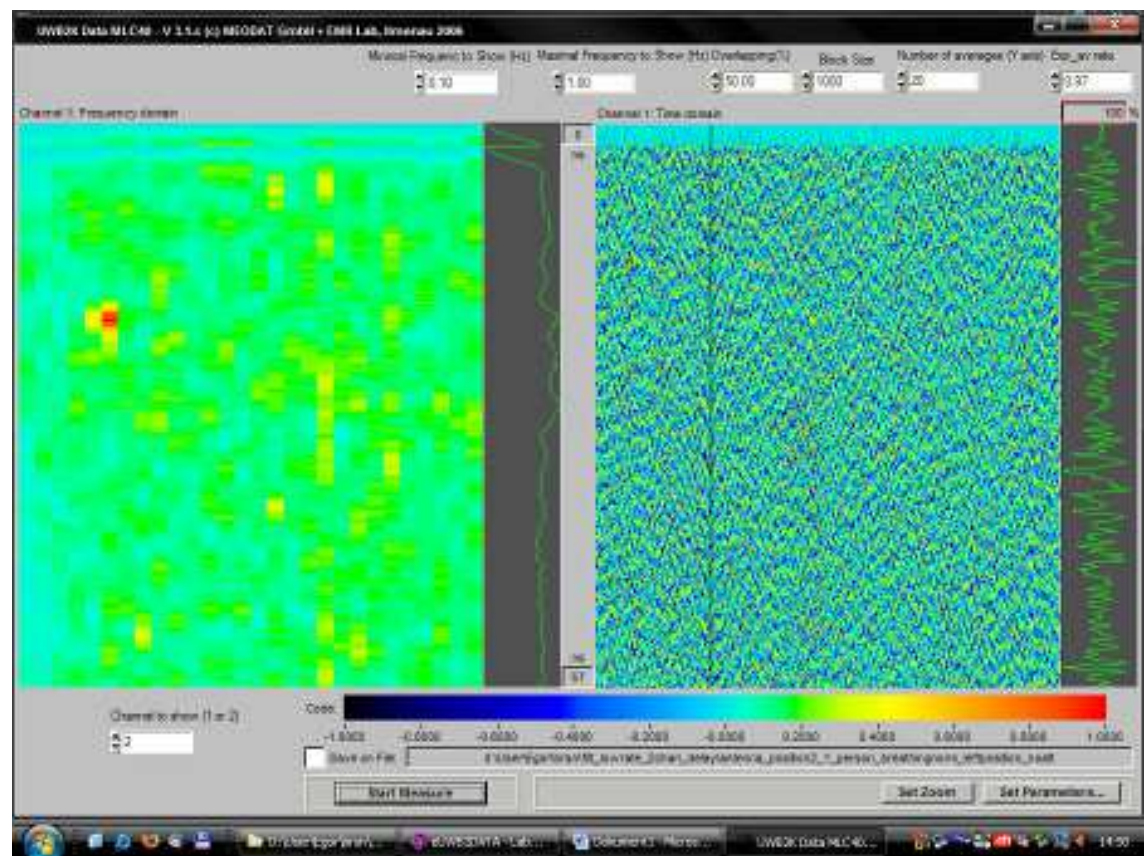

Fig. 17. One person detected through 1.2-1.3 meters of rubble

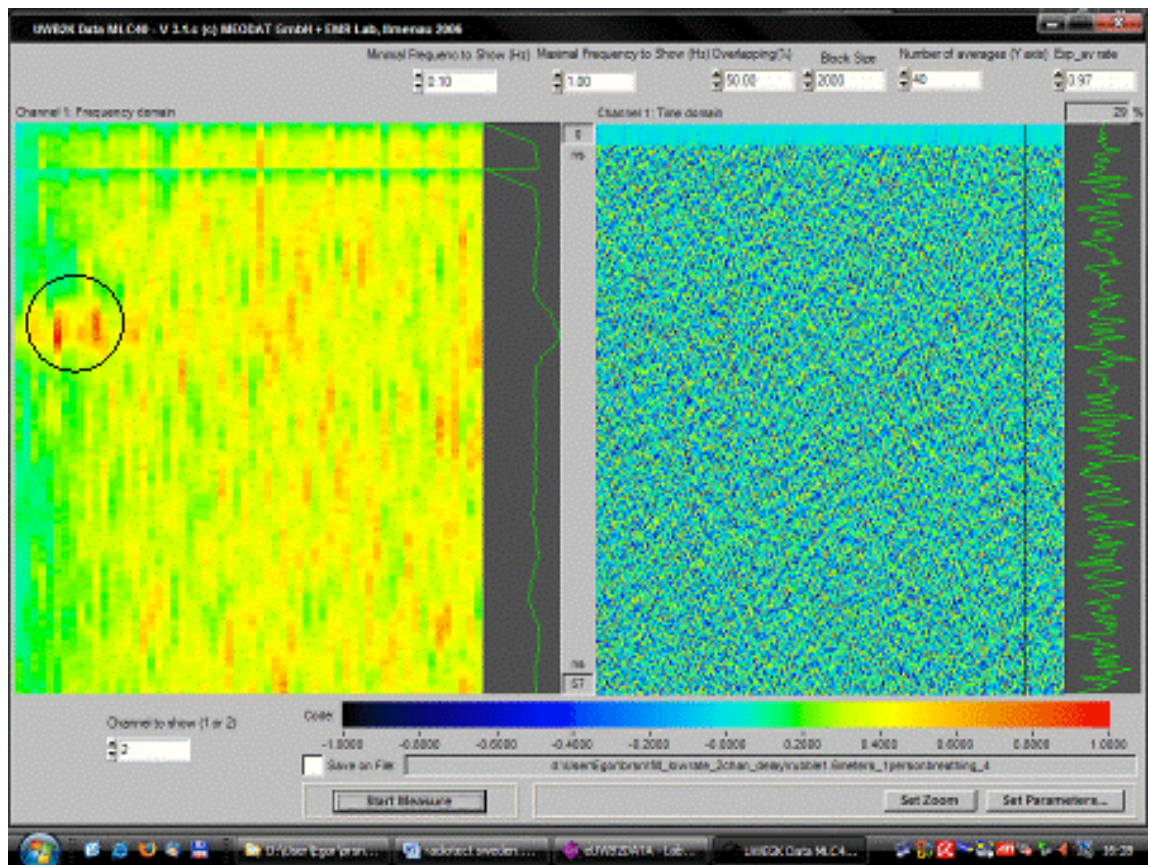

Fig. 18. One person detected through 1.6-1.7 meters of rubble 


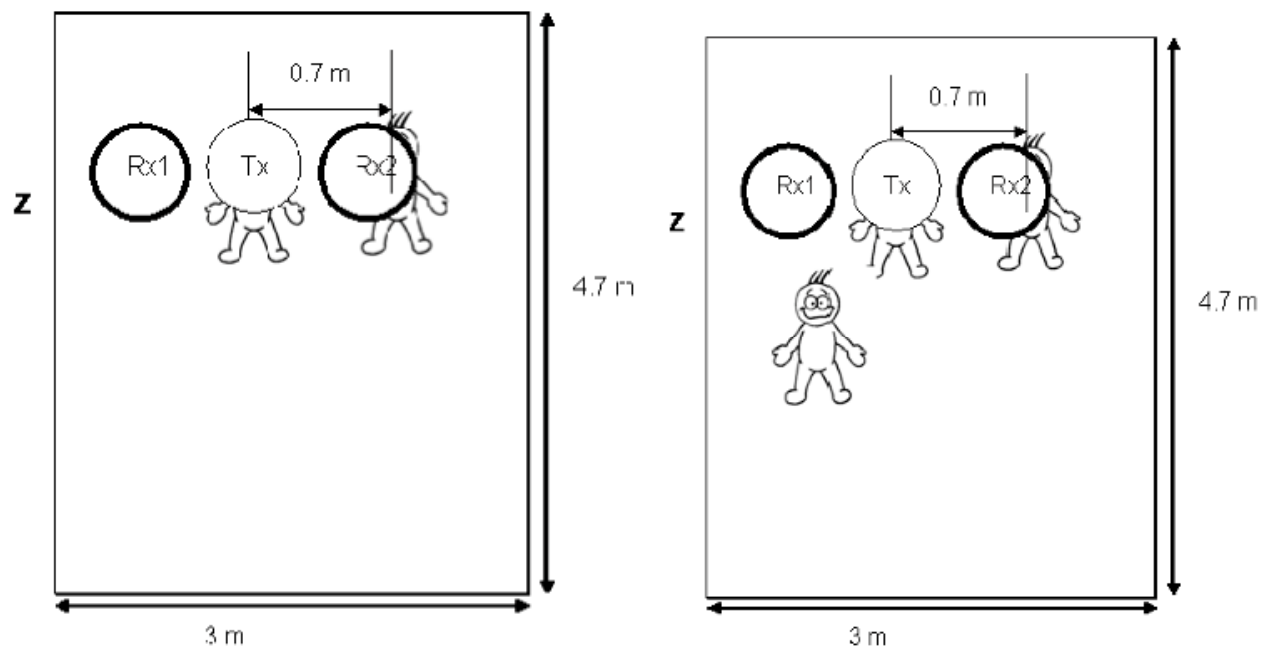

Fig. 19. Detection of multiple people through rubble pile, view from above. Identical positions as in scenario with two persons were used for $2 \mathrm{~d}$ localization in the next chapter.

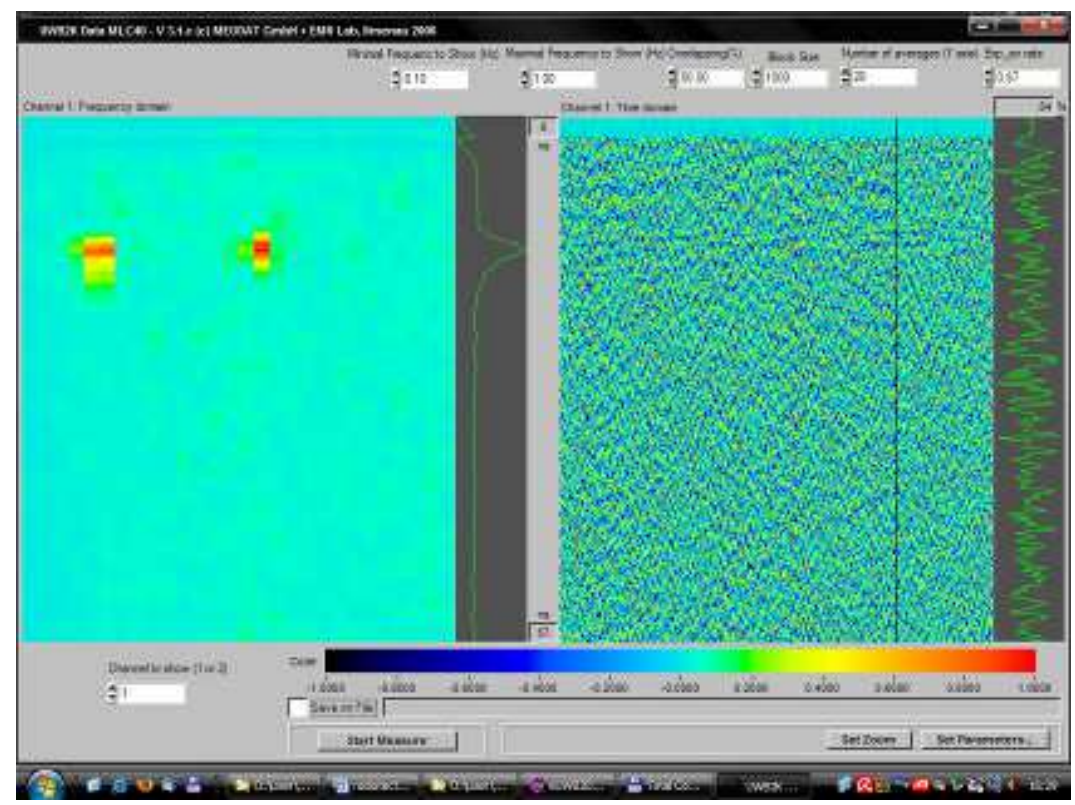

Fig. 20. Detection of two breathing persons through 1.2-1.3 meters of rubble 


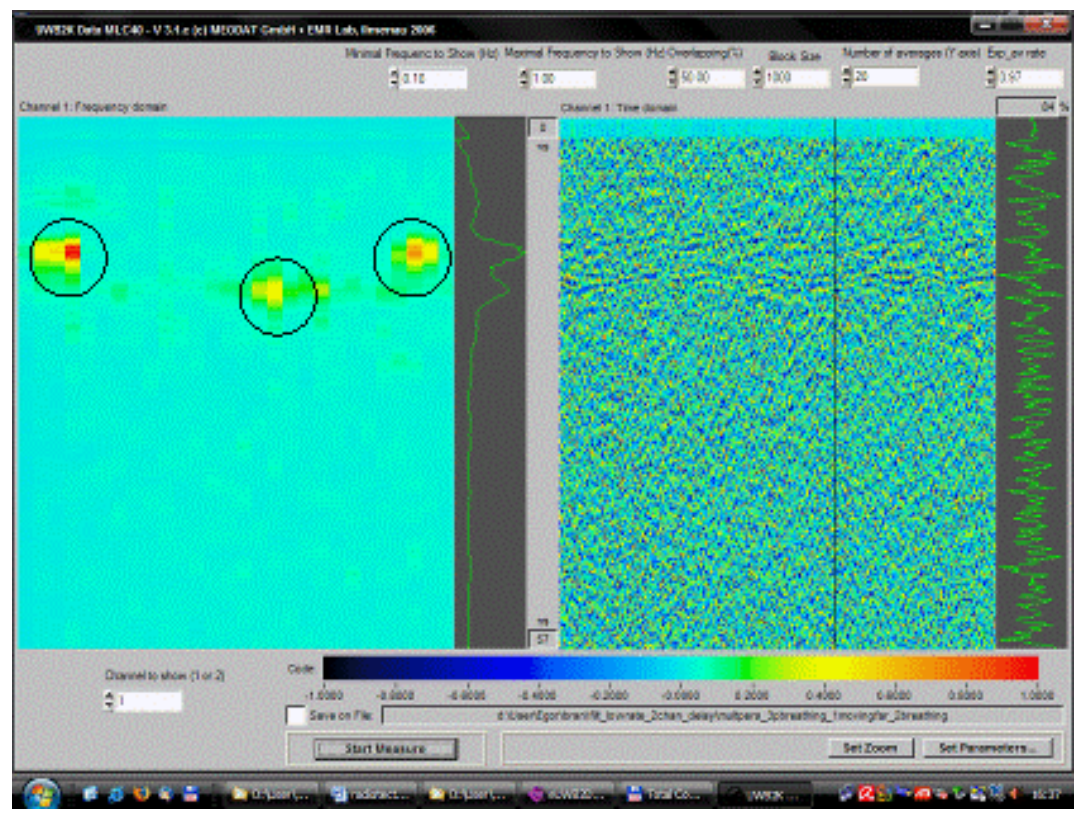

Fig. 21. Detection of three breathing persons through 1.2-1.3 meters of rubble. Person outside the plane containing antennas and other people is moving strongly with his hands.

\subsection{Victim localization}

Data collection for localization of victims was accomplished at the same heap of rubble as in the previous chapter. Two types of localization were successfully applied to measured data: $2 \mathrm{~d}$ localization when person is situated in the same plane as three antennas with combined TOA/TDOA method and $3 \mathrm{~d}$ localization of person with TOA when additional measurement after relocating antenna was carries out.
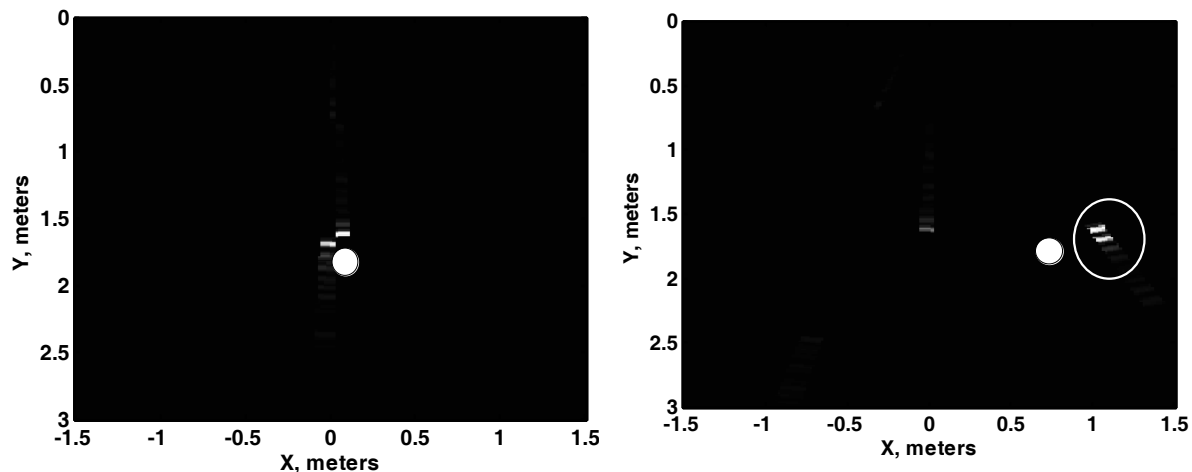

Fig. 22. Detection and $2 d$ localization of two persons breathing at different positions one after another (positions of persons and antennas are shown in figure 19). Pictures here represent vertical plane containing antennas and person. Zero of each axis corresponds to the centre of transmitting antenna. Actual positions of victims are shown as white circles. 

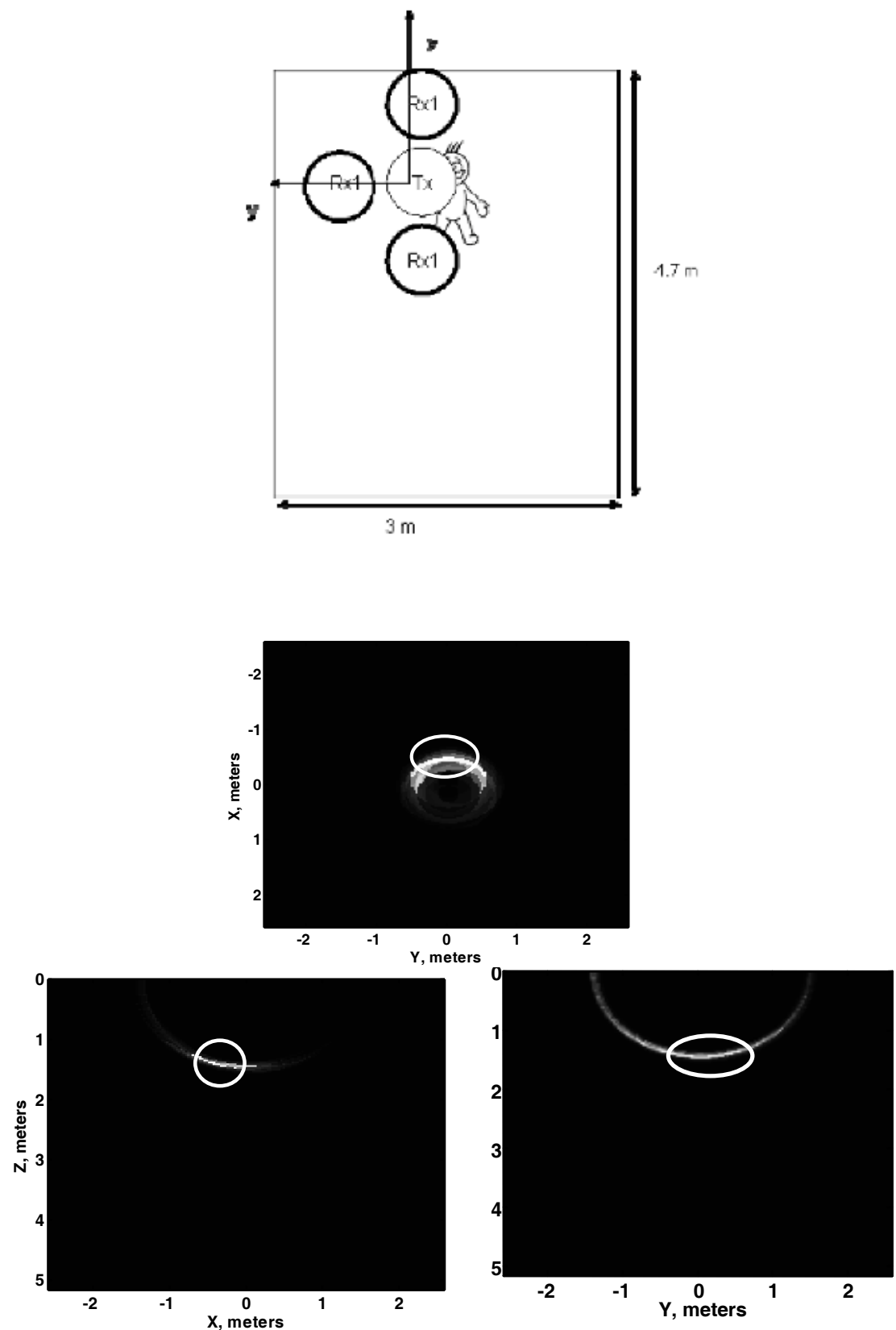

Fig. 23. Detection and $3 \mathrm{~d}$ localization of breathing person. Breathing is detected as maximum in $3 \mathrm{~d}$ array (marked). Zero of each axis corresponds to the centre of transmitting antenna. Actual position of victim is right beneath transmitting antenna. 


\section{Conclusions and future work}

Finally, we summarize the most important points of this chapter in relation to the possibilities of improving breathing-detecting radar further.

- In general, breathing enhancement is well developed from algorithmic point of view and results are reported in numerous papers. With our hardware and algorithms detection of breathing without any additional motion was possible through the maximum of $1.5-1.6$ meters of concrete rubble with reinforcements. Besides, ability to detect multiple victims is also shown. However, important target for future research is development of reliable detecting algorithm that provides information about victim presence automatically without operator.

- The problem of clutter removal is complex from algorithmic point of view because there is not much a priori information about unwanted signals that could serve as a basis for the solution. That is, cutter can overlap with breathing signature in distance, appearing in the same frequency range. Two approaches are demonstrated it this paper, but much more methods can be applied to this problem.

- Open questions in localization are 'ghost targets' as well as rubble heterogeneity. However, the problem of 'ghost targets' is not absolutely critical (it appears only when multiple victims are present at similar distance to antennas, and multiple victims can still be detected together with additional target). Furthermore, when victims have different breathing rates, this information can be used for separating real targets from 'ghosts'. Rubble heterogeneity can possibly be taken into account if some information about rubble structure is available (whether it is received by means of radar or any other method).

\section{References}

Arai, I. (2001). Survivor search radar system for persons trapped under earthquake rubble, Proceedings of APMC 2001, pp 663-668 vol.2, ISBN: 0-7803-7138-0, Taipei, Taiwan, December 2001

Bechtel Special Technologies Laboratory Ground Penetrating Radar (STL GPR) (2003). Operational Test and Evaluation Report of Ground Penetrating Radar for Casualty Location in Rubble, November 2003

BOS - Sondermaschinenbau GmbH. Locate to Live - Save of Live (Bioradar BR402)

Chernyak, V. (2006). Signal Processing in Multisite UWB Radar Devices for Searching Survivors in Rubble, Proceedings of EuRAD 2006, pp. 190 - 193, ISBN: 2-9600551-7-9, Manchester, September 2006

Geophysical Survey Systems, Inc. (2005). Technical Note 103: Using the SIR-3000 as a Breathing Detector with the "Breathe" Setup, February 2005

Levitas, B. \& Matuzas, J. (2006). UWB Radar for Human Being Detection Behind the Wall, Proceedings of IRS 2006, pp. 1 - 3, ISBN: 978-83-7207-621-2, Krakow, Poland, May 2006

Levitas, B. Matuzas, J. \& Drozdov, M. (2008). Detection and separation of several human beings behind the wall with UWB Radar, Proceedings of IRS 2008, pp. 1 - 4, ISBN: 978-83-7207-757-8, Wroclaw, Poland, May 2008

Narayanan, R.M. (2008). Through wall radar imaging using UWB noise waveforms, Proceedings of ICASSP 2008, pp. 5185 - 5188, ISBN: 978-1-4244-1483-3, Las Vegas, $\mathrm{NV}$, April 2008 
Ossberger, G.; Buchegger, T.; Schimback, E.; Stelzer, A. \& Weigel, R., Non-Invasive Respiratory Movement Detection and Monitoring of Hidden Humans Using Ultra Wideband Pulse Radar, Proceedings of the International Workshop on Ultrawideband Systems and Technologies, pp. 395-399, ISBN: 0-7803-8373-7, May 2004

UltraVision Security Systems, Inc. Product Backgrounder: LifeLocator ${ }^{\mathrm{TM}}$

Yarovoy, A.G. \& Ligthart, L.P. (2007). UWB Radars: Recent Technological Advances and Applications, Proceedings of Radar Conference, 2007 IEEE, pp. 43 - 48, ISBN: 1-42440284-0, Boston, MA, April 2007

Zaikov, E.; Sachs, J.; Aftanas, M.; \& Rovnakova J. (2008). Detection of trapped people by UWB radar, German Microwave Conference, GeMiC 2008, pp 240-243, ISBN: 978-38007-3086-5, Hamburg, Germany, March 2008

Zaikov, E.; (2010). UWB radar for detection and localization of trapped people, Proceedings of 11th International Radar Symposium IRS-2010, June 16-18, Vilnus, Lithuania, June 2010 


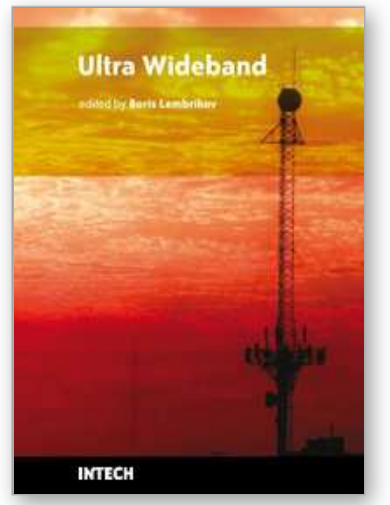

\author{
Ultra Wideband \\ Edited by Boris Lembrikov
}

ISBN 978-953-307-139-8

Hard cover, 458 pages

Publisher Sciyo

Published online 17, August, 2010

Published in print edition August, 2010

Ultra wideband technology is one of the most promising directions in the rapidly developing modern communications. Ultra wideband communication system applications include radars, wireless personal area networks, sensor networks, imaging systems and high precision positioning systems. Ultra wideband transmission is characterized by high data rate, availability of low-cost transceivers, low transmit power and low interference. The proposed book consisting of 19 chapters presents both the state-of-the-art and the latest achievements in ultra wideband communication system performance, design and components. The book is addressed to engineers and researchers who are interested in the wide range of topics related to ultra wideband communications.

\title{
How to reference
}

In order to correctly reference this scholarly work, feel free to copy and paste the following:

Egor Zaikov and Juergen Sachs (2010). UWB Radar for Detection and Localization of Trapped People, Ultra Wideband, Boris Lembrikov (Ed.), ISBN: 978-953-307-139-8, InTech, Available from:

http://www.intechopen.com/books/ultra-wideband/uwb-radar-for-detection-and-localization-of-trapped-people-

\section{INTECH}

open science | open minds

\section{InTech Europe}

University Campus STeP Ri Slavka Krautzeka 83/A 51000 Rijeka, Croatia

Phone: +385 (51) 770447

Fax: +385 (51) 686166 www.intechopen.com

\section{InTech China}

Unit 405, Office Block, Hotel Equatorial Shanghai No.65, Yan An Road (West), Shanghai, 200040, China 中国上海市延安西路65号上海国际贵都大饭店办公楼 405 单元 Phone: +86-21-62489820

Fax: +86-21-62489821 
(C) 2010 The Author(s). Licensee IntechOpen. This chapter is distributed under the terms of the Creative Commons Attribution-NonCommercialShareAlike-3.0 License, which permits use, distribution and reproduction for non-commercial purposes, provided the original is properly cited and derivative works building on this content are distributed under the same license. 